\title{
Tackling self-absorption in luminescent solar concentrators with type-II colloidal quantum dots
}

\author{
Zachar Krumer ${ }^{\mathrm{a}, *}$, Suzanne J. Pera ${ }^{\mathrm{a}}$, Relinde J.A. van Dijk-Moes ${ }^{\mathrm{a}}$, Yiming Zhao ${ }^{\mathrm{a}}$, Alexander F.P. de Brouwer ${ }^{\mathrm{a}}$, \\ Esther Groeneveld ${ }^{a}$, Wilfried G.J.H.M. van Sark ${ }^{b}$, Ruud E.I. Schropp ${ }^{c}$, Celso de Mello Donegáa \\ ${ }^{a}$ Condensed Matter and Interfaces, Debye Institute for NanoMaterials Science, Utrecht University, P.0. Box 80 000, 3508 TA Utrecht, The Netherlands \\ ${ }^{\mathrm{b}}$ Science Technology and Society, Copernicus Institute, Utrecht University, Budapestlaan 6, 3584 CD Utrecht, The Netherlands

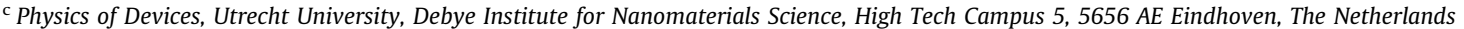

\section{A R T I C L E I N F O}

\section{Article history:}

Received 13 July 2012

Received in revised form

10 December 2012

Accepted 11 December 2012

Available online 20 January 2013

Keywords:

Luminescent solar concentrators

Self-absorption

Semiconductor hetero-nanocrystals

Quantum dots

Solar cells

\begin{abstract}
A B S T R A C T
Luminescent solar concentrators are low cost photovoltaic devices, which reduce the amount of necessary semiconductor material per unit area of a solar collector by means of concentration. The device is comprised a thin plastic plate in which luminescent species (fluorophores) have been incorporated. The fluorophores absorb the solar light and radiatively re-emit part of the energy. Total internal reflection traps most of the emitted light inside the plate and wave-guides it to a narrow side facet with a solar cell attached, where conversion into electricity occurs. The efficiency of such devices is as yet rather low, due to several loss mechanisms, of which self-absorption is of high importance. This work demonstrates that type-II semiconductor hetero-nanocrystals may offer a solution to the self-absorption problem in luminescent solar concentrators.
\end{abstract}

(c) 2013 Elsevier B.V. All rights reserved.

\section{Introduction}

Luminescent solar concentrators (LSCs) have been proposed as alternative photovoltaic (PV) devices, as they require less semiconductor material per unit of generated power, compared with conventional PV-technologies. While initially introduced as a means for better exploitation of high efficiency solar cells [1-3] and therefore for cost reduction, recently the focus has shifted towards LSCs as a promising concept for integration of PV into buildings $[4,5]$.

LSCs are essentially thin sheets of transparent plastics, in which luminescent species (luminophores) are dispersed (Fig. 1). Long and slim solar cells are attached to the small side faces such that their collection surface is directed to the plastic plate. Sun light enters the sheet through the large top face and is absorbed by the incorporated luminophores, which re-emit light. The wavelength of the re-emitted light is different from the incoming wavelength, just like the propagation direction. Because of this change in direction a large fraction of the re-emitted light cannot leave the plate through the plastic-air interface, as it is in the regime of total internal reflection (TIR). Therefore the light is wave-guided until it reaches the solar cell.

\footnotetext{
* Corresponding author. Tel.: + 31302533545

E-mail address: z.krumer@uu.nl (Z. Krumer).
}

This way the light entering a large face $A_{\text {top }}=a \cdot b$ is diverted onto a small face $A_{\mathrm{SC}}=b \cdot d$ of the solar cell. The maximum achievable geometric concentration factor for an LSC-device with one solar cell face and three faces with perfect mirrors is

$G=\frac{A_{\mathrm{top}}}{A_{\mathrm{SC}}}=\frac{a \cdot b}{b \cdot d}=\frac{a}{d}$,

where $a, b$ and $d$ are the length, width and thickness of the plastic sheet. The wavelength of the re-emitted light depends on the choice of the luminophore, which is ideally chosen such that the emission band matches the maximum of the spectral response of the solar cell. The solar cell will thus operate with a higher efficiency than under broad spectrum illumination [6,7].

The best, so far obtained efficiencies are still modest (7.1\% [8], $6.9 \%$ [9]), and have been obtained for rather small devices (a few $\mathrm{cm}^{2}$ ). The low efficiencies and limited dimensions of the currently available LSCs are due to a number of loss mechanisms. These loss mechanisms have been discussed in detail in the work of Roncalli et al. [10], and therefore will be only briefly addressed here. The optical loss mechanisms involved are partial surface reflection and parasitic absorption by the plastic sheet [11]. More significant losses occur due to non-radiative energy dissipation by the luminophores when their quantum yield $\eta_{\mathrm{LQE}}$ is low, as well as by light emission or reflection within the escape cone of the plastic-air interface, such that no TIR can take place [12]. For a single PMMA-air interface approximately $25 \%$ of the photons are 
thus lost (loss factor $P_{\text {conic }} \approx 0.25$ ) [2,13]. Further losses occur due to self-absorption. In this case, luminophores in the LSC re-absorb previously emitted photons before they can reach the solar cell. Upon every re-absorption event the photon energy can be dissipated again through one of the above mentioned loss channels, even if the re-absorbed photon was being transported in the wave-guiding mode towards the solar cell before the selfabsorption event occurred. Even in an idealised LSC with TIRescape losses only, the trapping probability for every emitted photon is $P_{\text {trap }}=1-P_{\text {conic }}=0.75$. Consequently for a given input energy flux $\Phi_{\text {in }}$ the output flux $\Phi_{\text {out }}$ decreases after an average of $N-1$ re-absorptions per photon to

$\Phi_{\mathrm{out}}=\Phi_{\mathrm{in}} \eta_{\mathrm{LQE}}^{N}\left(1-P_{\text {conic }}\right)^{N}$.

This equation shows that re-absorption maximises the occurring losses as the light output into the solar cell decreases exponentially with the number of re-absorption events [13]. This limits the size of LSC plates and thus the maximum concentration ratio. Therefore, it is essential to tackle the self-absorption problem in order to improve the efficiency of LSCs.

Re-absorption can be minimised by using luminophores that have a negligible absorption cross-section at their emission energy (Self-absorption cross-section: Section 2.2). This implies that their emission spectrum should be well separated from their absorption spectrum, i.e. the Stokes shift should be reasonably large. Among the luminophores with the required optical properties, colloidal type-II semiconductor hetero-nanocrystals are particularly promising. Semiconductor hetero-nanocrystals (HNCs) consist of two (or more) different materials joined in the same particle by hetero-interfaces. Depending on the energy offsets between the valence and conduction band levels of the materials that are combined at the hetero-interface, different carrier localisation regimes will be observed after photoexcitation (Fig. 2) [14]. In type-I HNCs both carriers are primarily localised in the same material, whereas in type-II HNCs electrons and holes are spatially separated, creating a spatially indirect exciton (see Section 2.1.2 below for details). The photon emitted upon

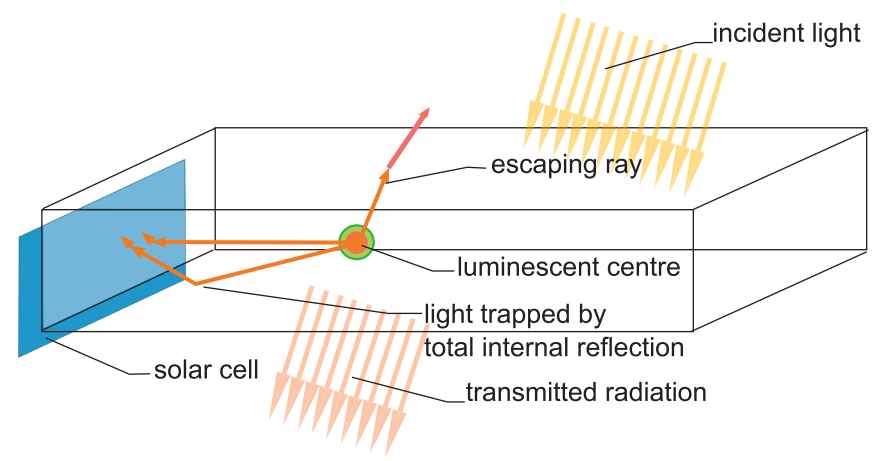

Fig. 1. Principle of operation of a LSC for building integrated PV.
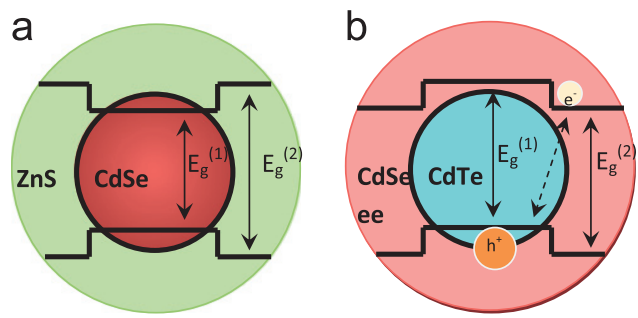

Fig. 2. Schematic representation of band structure in type-I QDs (left) and type-II semiconductor QDs (right). The spatially direct band gap energies are indicated as solid arrows. The spatially indirect one is shown as a broken line. radiative recombination of the spatially indirect exciton will have a lower energy than the band gap of both components of the HNC, and thereby its re-absorption probability is greatly reduced. TypeII HNCs have been successfully synthesised in recent years by a number of groups [14-17]. Recently, the suitability of type-II HNCs for application in LSCs has been theoretically investigated for the first time [18].

In this work, we compare the self-absorption behaviour of several types of widely applied luminophores (viz., three different organic dyes and CdSe QDs) with that of recently available CdTe/ CdSe type-II HNCs. In order to do so, we developed a liquid model LSC device, consisting of a quartz cell in which the studied luminophore solution can be quickly and easily exchanged. The loss mechanisms in this model LSC are the same as in a real LSC, and therefore our experiments offer a convenient and reliable way to evaluate the potential of luminophores for application in LSCs.

\section{Background}

\subsection{Materials}

\subsubsection{Dyes}

Organic luminescent dyes have been the first choice for LSCs, due to their high quantum yield and easy incorporation into PMMA or other polymer matrices. On the other hand, dyes emit in the visible range, which is at significantly higher energy than the band gap of silicon, thus leading to thermalisation losses in the silicon solar cell. A disadvantage from the perspective of LSCs is the usually narrow bandwidth of absorption for dyes, which results in conversion of only a fraction of the solar spectrum. This can be overcome, e.g., by the use of multiple LSC-plates, where dyes with different absorption spectra are incorporated [9]. Furthermore an additional limitation of dyes is that they frequently suffer from photo-degradation.

Rhodamine 6G has been suggested for application in LSCs already in the first publications for its high quantum yield (95\% $[1,19])$. The absorption band is however narrow. The Lumogen product group of BASF, which was specially designed for the application in LSCs [20], is commonly used as reference dye in LSCs [9,21-24]. Long-term outdoor studies have demonstrated its advantageous low degradation [12]. Moreover the overlap between absorption and emission is smaller than for other dyes such as Rhodamine 6G.

\subsubsection{Colloidal semiconductor nanostructures}

Colloidal semiconductor nanocrystals have also attracted a lot of interest as luminophores for LSCs. These nanocrystals are coated with a monolayer of organic molecules that render them easily dispersible in a variety of solvents, making solution processing of these materials extremely facile [14]. Moreover, quantum confinement effects become important for sufficiently small nanocrystals of direct band gap semiconductors. For this reason, colloidal semiconductor nanocrystals are typically referred to as colloidal quantum dots (QDs) or quantum rods, depending on their aspect ratio. In the strong quantum confinement regime the optical properties depend strongly on the nanocrystal size and shape, since the band gap becomes larger with decreasing nanocrystal size [25,14]. This effect can be exploited to adjust the emission wavelength of the QD to the maximum of the spectral response of the solar cell. QDs are ideal light harvesters and spectral converters for LSCs, since they have very broad absorption spectra (any photon with energy equal to or higher than the band-gap is efficiently absorbed) and narrow emission spectra [26]. Colloidal QDs can be easily produced by 
wet-chemical methods, achieving photoluminescence quantum yields as high as $80 \%[14,27]$.

The chemical stability of single composition QDs upon air exposure is typically low [14]. A commonly used strategy to improve the stability of QDs is coating with a shell of a wider band gap semiconductor, in such a way that a type-I heteronanocrystal (HNC) is obtained (e.g., by growing a ZnS shell over a CdSe core QD) [14]. Colloidal type-I QDs have been shown to be more stable against photo-degradation than dyes [21], which is an important prerequisite for long-term light harvesting. A number of recent publications have demonstrated the application of colloidal type-I QDs as luminophores for LSCs [26,28-30]. However, the Stokes shift for single composition QDs and type-I HNCs is very small ( $<20 \mathrm{meV}$ ) [14]. The efficiency of LSCs based on type-I HNCs is thus limited by self-absorption losses. From this perspective, the recently introduced type-II HNCs (Fig. 2) are promising fluorophores for LSCs. As mentioned in the Introduction, the self-absorption cross-section of type-II HNCs is expected to be negligible, due to the large spectral separation between the emission peak and the lowest energy absorption edge $[14,31,32,16]$. Type-II colloidal HNCs have been realised for a number of compositions (e.g., $\mathrm{CdTe} / \mathrm{CdSe}, \mathrm{ZnSe} / \mathrm{CdS}$, etc.) $[14,31,32,16]$. In this study we focus on CdTe/CdSe HNCs, since high quality colloidal HNCs of this composition have become recently available $[31,17,33]$.

\subsection{Self-absorption cross-section}

Self-absorption occurs when there is a finite probability for a luminophore to absorb the light emitted by the same species. This is visualised in an overlap between the spectra of absorption $A(\lambda)$ and emission $F(\lambda)$. This overlap $\sigma_{\mathrm{SA}}$ quantifies the self-absorption cross-section per $1 \mathrm{~cm}$ optical path as

$$
\begin{array}{r}
\sigma_{\mathrm{SA}}=\frac{1}{K} \int_{0}^{\infty} F(\lambda) A(\lambda) \mathrm{d} \lambda \\
\text { with } K=\int_{0}^{\infty} F(\lambda) \mathrm{d} \lambda .
\end{array}
$$
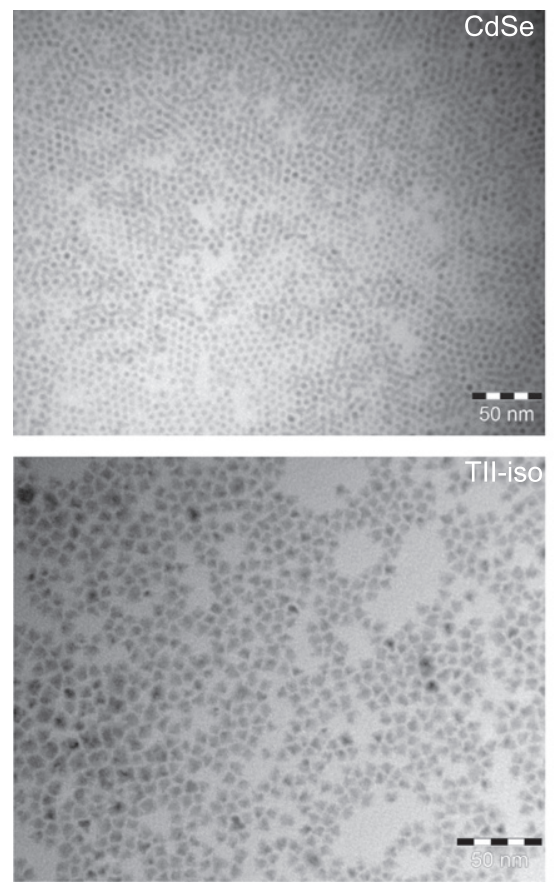

This expression gives the fraction of the emission that is absorbed by the luminophore itself while travelling through $1 \mathrm{~cm}$ of the medium. This optical path-length was chosen because it corresponds to that used for the absorption measurements in solution. The integration is carried out between the boundaries of the wavelength in the emission spectrum. The self-absorption crosssection can be used to characterise luminescent species from their emission spectra and absorption spectra.

\section{Materials and methods}

\subsection{Materials}

The dyes used for the experiment were purchased as powders. Rhodamine 6G was obtained from Radiant Dyes Laser \& Accessories $\mathrm{GmbH}$ dissolved with absolute ethanol for experiments. Lumogen Orange and Lumogen Red were supplied by Kremer Pigmente as "94738 Fluoreszenzorange" and "94720 Fluoreszenzrot" and used as solutions in anhydrous toluene. Colloidal CdSe QDs were synthesised following the method reported by Peng et al. [15]. Colloidal type-II CdTe/CdSe HNCs with two different shapes were investigated: concentric $\mathrm{CdTe} / \mathrm{CdSe} / \mathrm{ZnS}$ core/multishell QDs (hereafter labelled TII-iso) and CdTe/CdSe dot core/rod shell hetero-nanorods (hereafter labelled TII-an). The TII-iso typeII QDs were synthesised following a method introduced by Zhang et al. [17]. The method described by Zhong and Scholes [33] was used to synthesise the TII-an type-II nanorods. Silica nanoparticles were synthesised for reference scattering measurements following the method described by Koole et al. [34]. Representative transmission electron microscopy (TEM) images of the nanoparticles synthesised in this work are provided in Fig. 3.

\subsection{Optical characterisation}

Absorption spectra of solutions of the luminophores were recorded with a Perkin-Elmer double beam UV/Vis spectrophotometer, using the solvent as the reference. The amount of
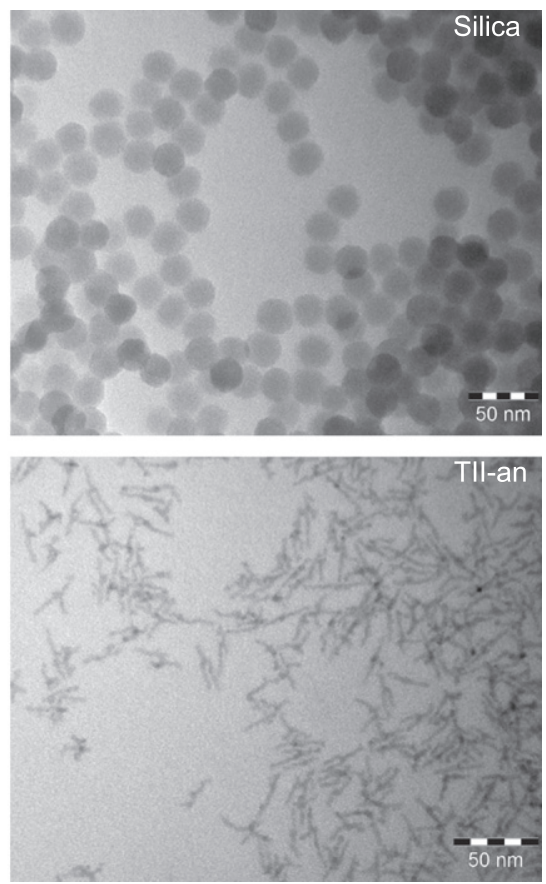

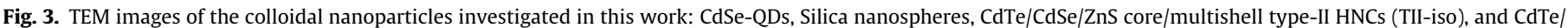
CdSe type-II dot-core/rod-shell nanorods (TII-an). 
scattered light was negligible in all cases. Therefore, the absorption $(A)$ could be obtained directly from the measured optical densities $(\alpha)$ as $A=1-10^{-\alpha}=1-T$. The quantum yield was measured with an integrating sphere using sufficiently diluted samples (sub-microM concentration range). The setup was an Edinburgh Instruments spectrofluorimeter with double-grating excitation and emission monochromators, and a $450 \mathrm{~W}$ Xenon lamp as the excitation source. Colloidal QDs and HNCs were excited with a xenon lamp at $\lambda_{\mathrm{ex}}=400 \mathrm{~nm}$, Lumogen Orange at $\lambda_{\mathrm{ex}}=450 \mathrm{~nm}$. The intensity of the excitation light was measured in the domain of the excitation wavelength $<\lambda \pm 5 \mathrm{~nm}>$ after it had passed the sample $<\mathrm{T}_{\text {sample }}(\lambda)>$ and after it had passed the solvent only $<\mathrm{T}_{\text {solvent }}(\lambda)>$. After the recorded spectra were corrected by the sensitivity function $(S(\lambda))$ of the detector, the difference of the two intensities was attributed to absorption. The emission spectra were measured under the same conditions as the absorption, but detected over a broader spectral region starting at a wavelength $5 \mathrm{~nm}$ longer than that used for excitation. The emission spectra were recorded for the excited sample solution $\left(F_{\text {sample }}(\lambda)\right)$ and for the solvent only $\left(F_{\text {solvent }}(\lambda)\right)$ and subsequently corrected. Integrating the emission and absorption as

$F=\int_{\lambda_{\text {ex }}+5 \mathrm{~nm}}^{\infty} \frac{F_{\text {sample }}(\lambda)-F_{\text {solvent }}(\lambda)}{S(\lambda)} \mathrm{d} \lambda$,

$A=\int_{\lambda_{\mathrm{ex}}-5 \mathrm{~nm}}^{\lambda_{\mathrm{ex}}+5 \mathrm{~nm}} \frac{T_{\text {solvent }}(\lambda)-T_{\text {sample }}(\lambda)}{S(\lambda)} \mathrm{d} \lambda$,

results in the luminescence quantum yield:

$\eta_{\mathrm{LQE}}=\frac{F}{A}$.

\subsection{Intensity profiles}

The measurements of self-absorption effects were conducted with a position-adjustable excitation source that illuminates different spots in the material so that the optical path length travelled by the emitted light inside the luminophore solution can be varied (Fig. 4). A Spectral Products ASBN-D2-W100F-L Halogen lamp (power $100 \mathrm{~W}$, colour temperature $3000 \mathrm{~K}$ ) filtered through a band filter at $(520 \pm 25) \mathrm{nm}$ was used as excitation source. The use of narrow-band light for excitation was necessary to allow luminophores with widely different absorption spectra to be reliably compared. The optical densities of the luminophore solutions were adjusted to $0.17 \pm 0.04$ at $520 \mathrm{~nm}$, in order to ensure that the amount of light absorbed by different samples was comparable (see spectra in supplementary information). However, it should be noted that the effective photon absorption cross-section for different luminophores could not be rendered identical, since the excitation bandwidth $\pm 25 \mathrm{~nm}$ was not sufficiently narrow to prevent variations due to the different absorption band shapes. Nevertheless, these variations have no impact on the validity of the conclusions drawn from the experiments, since we compare only the fraction of the emitted light that is lost due to self-absorption, and not the absolute intensities.

An optical fibre with a slit exit aperture and a lens was used to focus the excitation light on a quartz cell (the liquid model LSC device) (Fig. 4). The quartz cell was screw capped to prevent oxidation of the luminophore solution inside. The internal volume of the cell was $10 \mathrm{~mm} \times 10 \mathrm{~mm} \times 35 \mathrm{~mm}$. The bottom of the quartz cell was mounted in firm contact with a second optical fibre, which collected the emitted light and guided it to a monochromator and subsequently to a CCD (Spectra Pro 300i from Action Research) to record the emission spectra. The cell with the collection fibre could be moved along the $x$-axis with a step-size of several micrometres. When operating with airsensitive samples, the cells were filled under nitrogen atmosphere. The $x$-axis was chosen to point upwards to collect eventual gas bubbles in the bottleneck of the quartz cell, such that they could not influence the measurement. Toluene was employed as solvent for all the samples but Rhodamine 6G, which was dissolved in Ethanol.

\section{Results and discussion}

\subsection{Characterisation}

The absorption and emission spectra are provided in Fig. 5. The self-absorption cross-section of Rhodamine 6G was calculated to be $8.5 \%$ and its quantum yield was taken to be $95 \%$ [19]. The quantum yield of Lumogen Orange was found to be $95 \%$. The selfabsorption cross-section was calculated to be $6.7 \%$. The absorption spectra of the semiconductor QDs (Fig. 5) are much wider than those of the dyes, since they consist of a large number of different exciton transitions that partially overlap producing a spectrally broad absorption spanning from the band edge to the UV. The self-absorption cross-sections of the semiconductor QDs were calculated to be $52.2 \%, 2.5 \%$, and $0.2 \%$, for CdSe, type-II-iso, and type-II-an QDs, respectively. The quantum yields of the semiconductor QDs were found to be $27 \%$ and $48 \%$, for CdSe and type-II-iso QDs, respectively. The absorption and emission spectra of type-II-an QDs are similar to those of type-II-iso QDs, and are shown in the supplementary information.

\subsection{Self-absorption effects}

The emitted light can subsequently be absorbed by the emitting medium itself. As the Lambert-Beer law states, the probability for absorption grows exponentially with increasing path the light has to travel through the medium. The longer the path between the spot of excitation and the detector, the higher the probability for this process to occur and to repeat itself. In a

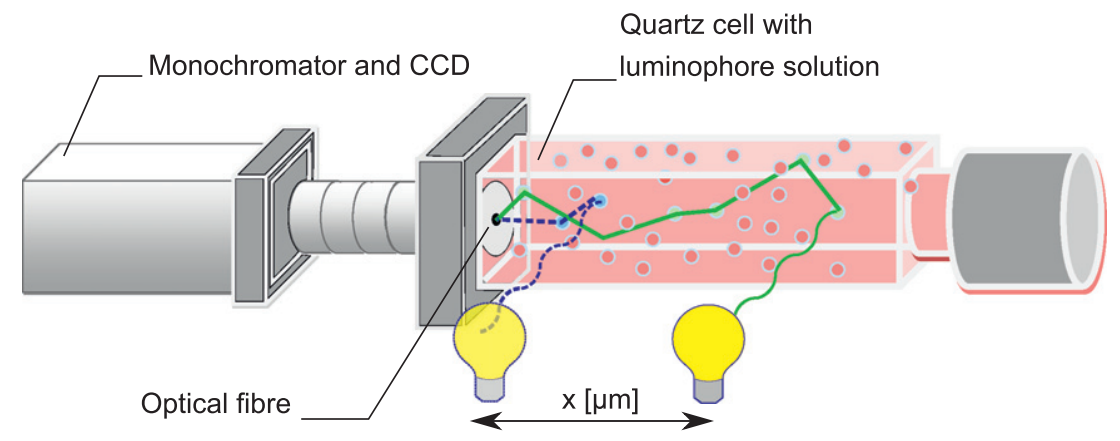

Fig. 4. Schematic representation of the setup for intensity profile measurements. 


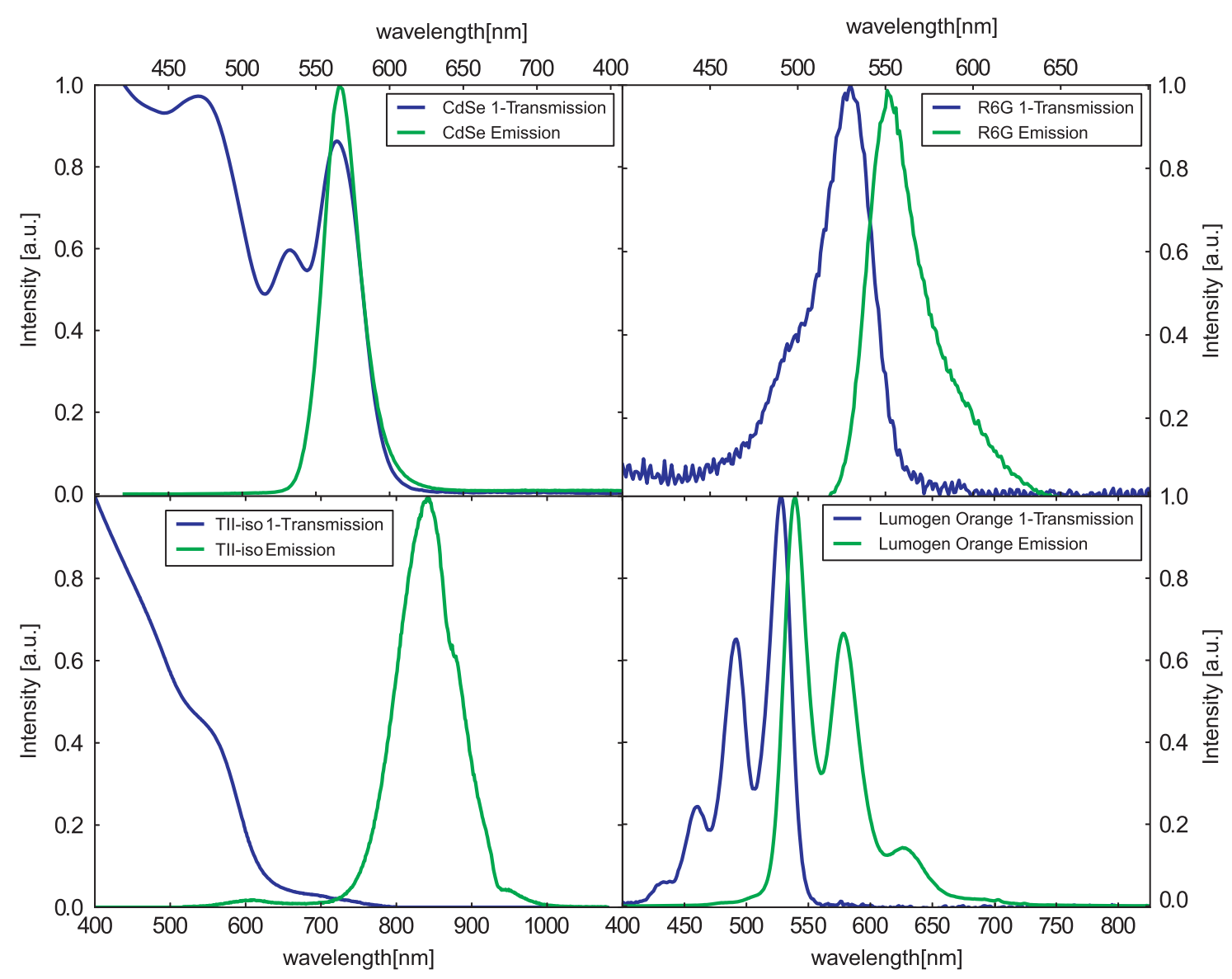

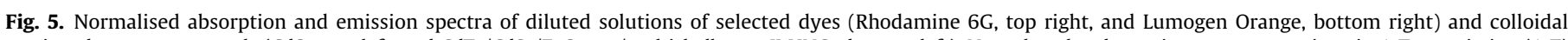

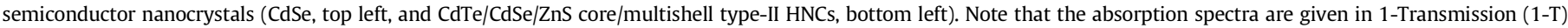
scale.

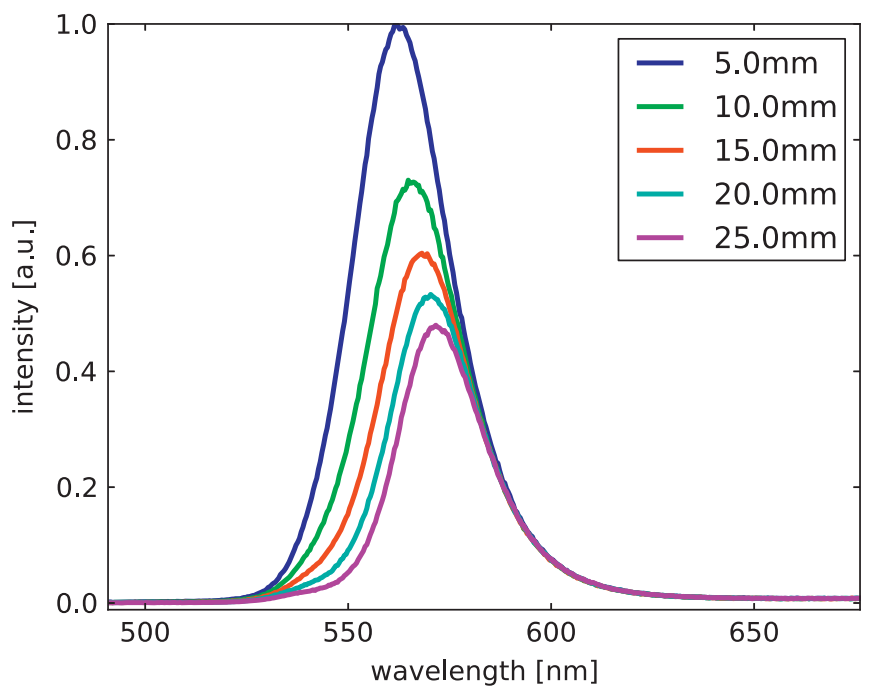

Fig. 6. Emission spectra of CdSe quantum dots recorded at different distances from the excitation spot.

weakly luminescent sample, such as the CdSe QDs investigated here (Fig. 6), the measured emission peak-intensity drops rapidly with increasing optical path. Although a spectral shift of the peak is observed, the position of the red band edge of the peak remains unchanged.

For single composition QDs (e.g., CdSe QDs) and type-I HNCs the energy of every emitted photon is up to a few $(<20) \mathrm{meV}$ lower than the band gap of the emitting quantum dot [14,35,36]. This is the intrinsic (or resonant) Stokes-shift. The so-called global (or non-resonant) Stokes-shift is larger and its origin is found in the fact that real samples always consist of an ensemble of QDs of slightly different sizes. The size distribution of the ensemble of QDs is reflected in the line width of the optical transitions, which becomes broader due to inhomogeneous broadening, and in the non-resonant Stokes shift, which increases for larger size dispersions and higher concentrations [36]. The increase in the nonresonant Stokes shift is due to the fact that the absorption crosssections at energies far above the band-edge scale with the volume [37]. Therefore, larger QDs will absorb relatively more light, resulting in a red-shift of the ensemble PL spectrum from the statistically weighted maximum at excitation energies far above the band-edge (such as those used in the present work). Moreover, self-absorption also leads to a concentration- and optical path-length-dependent increase in the observed nonresonant Stokes shift. Fig. 6 shows that this increase is primarily due to the shift of the emission peak to lower energies with increasing number of self-absorption events, which can occur as a result of increasing optical path lengths (Fig. 6) and/or increasing concentrations. A requirement for the self-absorption process is that the photon energy must be equal to or larger than the band gap of the absorbing QD. Therefore, photons emitted by the largest QDs in the ensemble do not have sufficient energy to be re-absorbed by most of the QDs in the ensemble, and therefore have a higher probability of reaching the detector. In contrast, photons emitted by smaller QDs carry sufficient energy to be absorbed by most members of the QD-ensemble and therefore are 


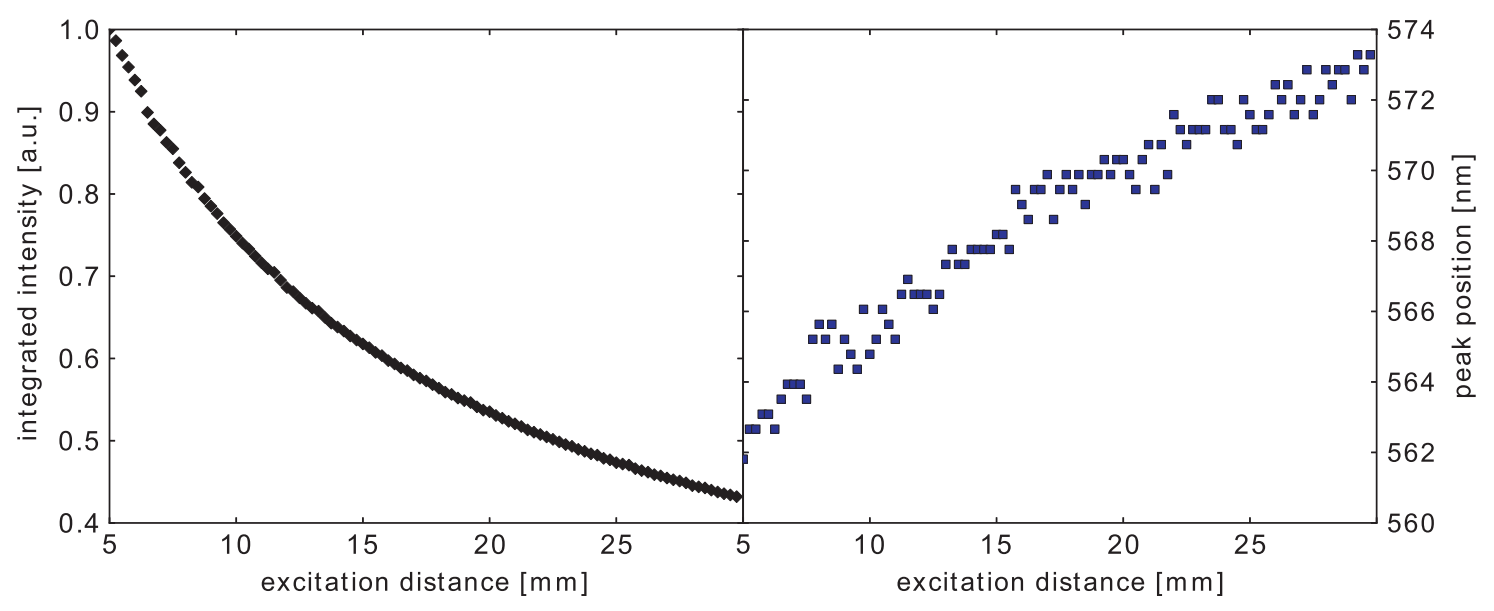

Fig. 7. Effects of self-absorption on intensity (left) and on peak position (right) of colloidal CdSe QDs.

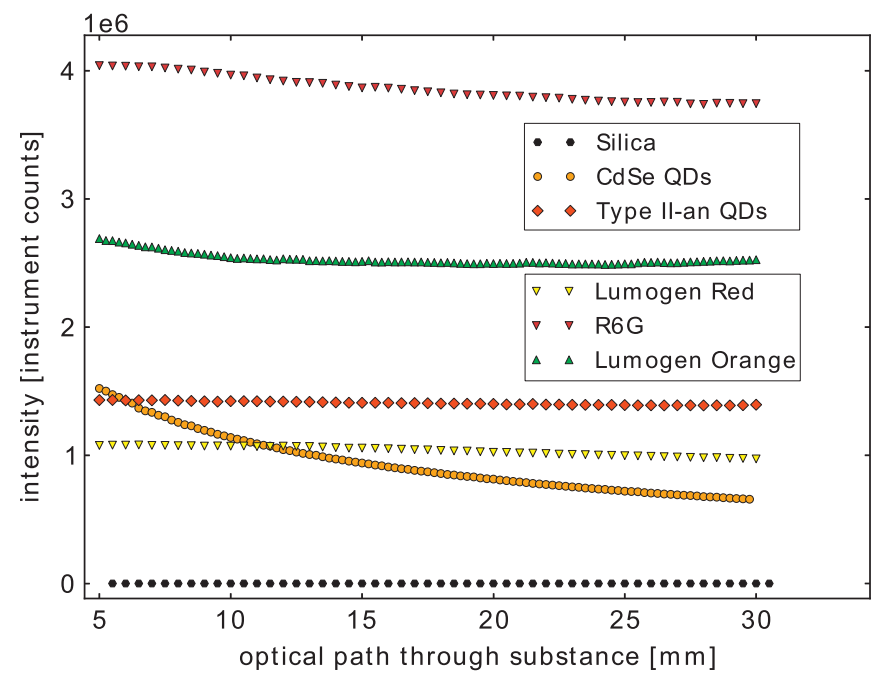

Fig. 8. Absolute intensity profiles for CdSe QDs, type-II-an QDs, Lumogen Orange, Lumogen Red, Rhodamine 6G, and silica nanoparticles. Note that the signal for silica nanoparticles is totally due to scattering of the excitation light, since these particles are non-luminescent.

more likely to be absorbed before reaching the detector. This is consistent with the observed loss in intensity in the range 530$570 \mathrm{~nm}$ and the almost unchanged red shoulder starting from $580 \mathrm{~nm}$, as the optical path increased (Fig. 6). The overall effect is the loss in total intensity and a total red-shift as shown in Fig. 7 for CdSe QDs.

The intensity decrease appears exponential (Fig. 7, left). Nevertheless the behaviour cannot be strictly exponential, because the fraction of absorbed photons is not constant. It depends on the wavelength of the input photons, which changes upon every selfabsorption event.

The integration of the recorded emission spectra provides a measure for the amount of light that would reach the solar cell at the side-face of a luminescent solar concentrator. We have performed the same analysis on a selection of dyes and colloidal semiconductor QDs and HNCs. The same experiments were also carried out on non-luminescent silica nanoparticles of comparable size $(25 \mathrm{~nm})$ to the QDs $(5-15 \mathrm{~nm})$ in order to provide a reference for potential losses due to light scattering. The results show that the impact of light scattering is negligible (Fig. 8, black line).

Although the optical coupling throughout the measurement of one sample is constant, that cannot be guaranteed when the sample is changed, since small variations in the optical alignment between the cuvette and the in-coupling and out-coupling fibres may occur. Moreover, the quantum yields and absorption band shapes within the wavelength range of the excitation light are not the same for the different luminophores. Therefore, in order to allow the self-absorption characteristics of the different samples to be properly compared, we normalised the intensities with respect to that acquired at the closest distance $(d=5 \mathrm{~mm})$ from the detector (Fig. 9).

All samples show intensity losses over the first half of the optical path. The observed losses are summarised in Table 1 . The quantitative differences allow the classification of the investigated luminophores in three distinct groups: those with a strong intensity loss (group A: CdSe QDs), those for which the output is moderately reduced (group B: Lumogen and Rhodamine dyes), and those for which the output is only slightly reduced (group C: both type-II HNCs). The increase in intensities at longer optical paths observed in some cases can be explained by the increasingly stronger reflection of excitation light as the bottleneck of the quartz cell is approached.

\subsection{Red-shift}

As discussed above for CdSe QDs, the increase of the optical path length travelled by the emitted light prior to detection leads to a red-shift of observed emission peak (Figs. 6 and 7). However, the magnitude of the red-shift is not the same for different luminophores, as can be clearly seen in the emission spectra of the type-II-iso QDs (Fig. 11) and Lumogen Orange (Fig. 10). To allow the different luminophores to be compared the red-shift $\Delta \lambda$ is calculated as

$\Delta \lambda=\lambda_{\max }\left(d_{\max }\right)-\lambda_{\max }\left(d_{\min }\right)$,

where $\lambda_{\max }(d)$ denotes the wavelength at which the emission attains its maximum. The argument $d$ is the length of the optical path at which the spectrum was recorded.

The observed intensity loss in groups A and B can be confidently attributed to self-absorption as it is accompanied by a red-shift of the peak (Fig. 12). This red-shift is also correlated to the intensity drop: the luminophore with the strongest intensity loss (CdSe QDs) also reveals the largest red-shift (and lowest quantum yield) (see Table 1). The spectra of the group B are only slightly red-shifted, however, with a well pronounced trend to increase.

\subsection{Type-II QDs in comparison with other luminophores}

For type-II HNCs a red-shift can be formally calculated with Eq. (7). However, facing the relatively strong data scattering 
a

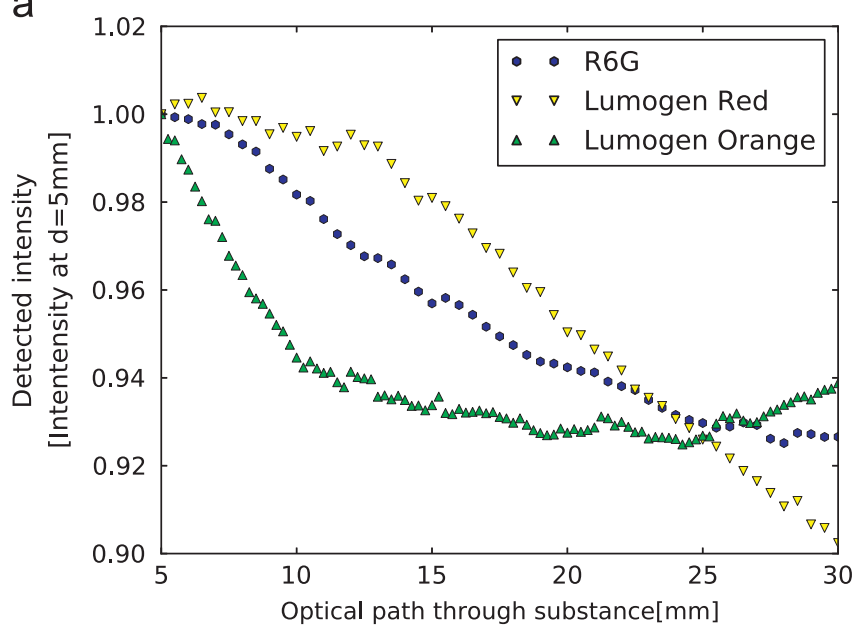

b

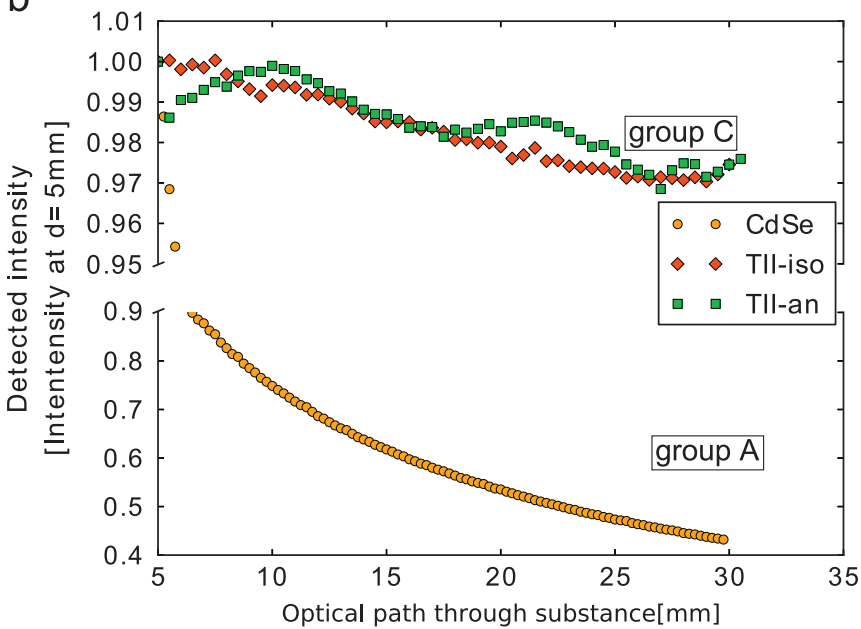

Fig. 9. Normalised intensity profiles of a selection of dyes (left) and semiconductor nanocrystals (right).

Table 1

Summary of the key quantities determined in the present work for a number of luminophores: CdSe QDs, Rhodamine 6G (R6G), Lumogen Orange (Lumo O), CdTe/ CdSe/ZnS-core/multishell type-II QDs (TII-iso), and CdTe/CdSe dot-core/rod-shell type-II nanorods (TII-an): $\eta_{\mathrm{LOE}}$, luminescence quantum yield; $\sigma_{\mathrm{SA}}$, self-absorption cross-section; $\Delta \lambda$, spectral shift after $30 \mathrm{~mm}$ optical path length; Loss, relative intensity loss after $30 \mathrm{~mm}$ optical path length; and $\xi$, integrated absorption spectra from 300 to $800 \mathrm{~nm}$ normalised with respect to Lumogen Orange.

\begin{tabular}{llccll}
\hline Sample & $\eta_{\text {LEE }}(\%)$ & $\sigma_{\text {SA }}(\%)$ & $\Delta \lambda(\mathrm{nm})$ & Loss $(\%)$ & $\xi$ \\
\hline CdSe QDs & 27 & 52.2 & 11.0 & 57 & 4.04 \\
R6G & 95 & 8.5 & 4.0 & 7.0 & 1.31 \\
Lumo O & 95 & 6.7 & 2.2 & 7.5 & 1.0 \\
TII-iso & - & 2.5 & -0.6 & 3 & 4.13 \\
TII-an & 48 & 0.2 & -0.6 & 3 & 4.02 \\
\hline
\end{tabular}

(Fig. 12) it is more meaningful to consider the mean value of the shift, which is $-0.6 \mathrm{~nm}$ for both type-II HNC samples (Table 1 ). This average is of the same order of magnitude as the resolution of the monochromator used $(0.5 \mathrm{~nm})$ and hence a strong indicator for no red-shift at all. Together with the minimal intensity loss this indicates the absence of self-absorption in type-II samples.

The long wavelength part of the emission spectra of the type-II HNCs could not be recorded accurately as the sensitivity of the used CCD is very low beyond $860 \mathrm{~nm}$ (Fig. 11). Since the intensity drops mostly at the blue end of the spectrum eventual self-absorption effects could be detected if present. The red

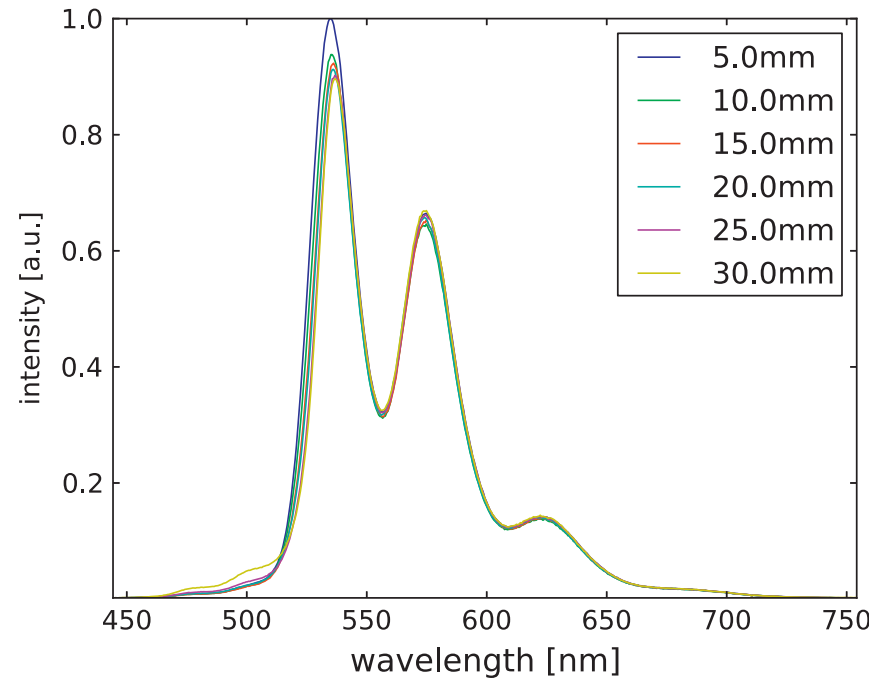

Fig. 10. Emission spectra of Lumogen Orange recorded at different distances from the excitation spot.

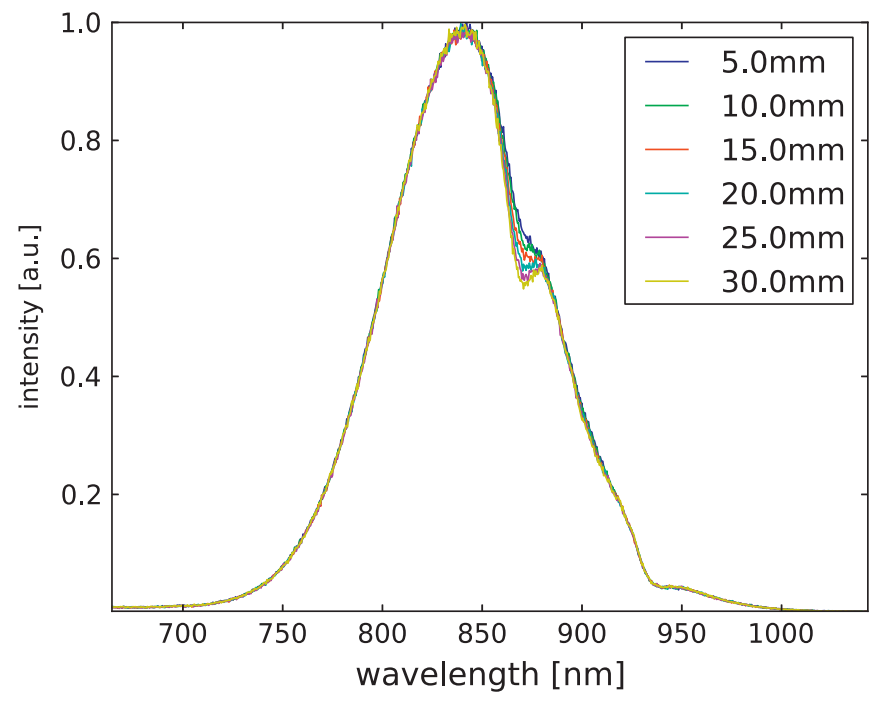

Fig. 11. Emission spectra of type-II-iso quantum dots recorded at different distances from the excitation spot.

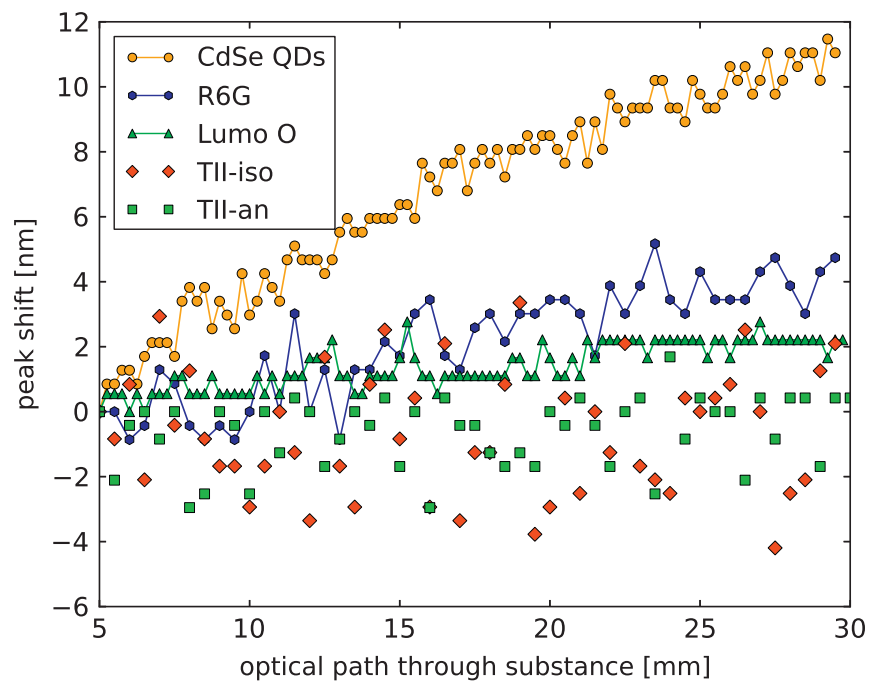

Fig. 12. Shift of maximum of the emission band with increasing optical path of a selection of luminophores related to the initial position. 
$(\lambda>860 \mathrm{~nm})$ part of the spectrum is not entirely reliable. This inaccuracy means that the intensities reported here for TII-iso and TII-an QDs represent a lower limit, and the overall output flux might be larger.

From all the investigated samples the type-II HNCs show the least self-absorption effects. Even if compared with the dye with the best performance (Lumogen Orange, Fig. 10) the photons emitted by the type-II HNC samples seem almost unaffected by the amount of material they travelled through. It should be pointed out that monochromatic excitation was used in our experiments. Although these conditions are suitable to allow the self-absorption properties of different luminophores to be reliably compared, they do not reveal an important advantage of colloidal semiconductor NCs: their broad absorption spectrum. In order to account for the total amount of light that could potentially be absorbed by the different luminophores under excitation by a broad band source (like the sun) we have integrated their absorption spectra from 300 to $800 \mathrm{~nm}$, and normalised the result with respect to the total absorption crosssection of Lumogen Orange (quantity $\xi$ in Table 1). The amount of emitted light $F$ is then given by

$F=\xi \cdot \eta_{\mathrm{LQE}}$

If this is taken into account one can conclude that under uniform broad band excitation the light output of type-II-iso HNCs at $1 \mathrm{~cm}$ optical path length would be $\sim 2$ times larger than that of the Lumogen Orange, despite its lower PL QY (48\% vs. 95\%, see Table 1). However, PL QYs as high as $80 \%$ have been reported in the literature for CdTe/CdSe type-II HNCs similar to those investigated in the present work [36], demonstrating that the high outputs reported here for type-II HNCs can be even better with proper optimisation of the sample preparation.

\section{Conclusions}

In this work, we have compared the self-absorption behaviour of a number of luminophores in a model liquid LSC: colloidal CdSe QDs, Rhodamine 6G, Lumogen Orange, Lumogen Red, and colloidal $\mathrm{CdTe} / \mathrm{CdSe}$ type-II hetero-nanocrystals (HNCs). Our results clearly demonstrate that the re-absorption losses of type-II CdTe/ CdSe HNCs are negligible with respect to the other luminophores investigated. This shows that type-II colloidal QDs are promising materials for Luminescent Solar Concentrators, as they have the potential to strongly minimise the efficiency losses induced by self-absorption. Moreover, their emission in the near infra-red $(900 \mathrm{~nm})$ is of high importance for application in LSCs, since it matches the region of maximal spectral response of crystalline silicon solar cells. In this spectral range, organic dyes do not perform well, due to a combination of lower quantum yields ( $\approx 50 \%$ or below [38]) and poor photostability.

Furthermore, the broad absorption spectra of these colloidal HNCs make them excellent light harvesters, which should further increase the potential light output of LSCs. The quantum yield of the type-II HNC samples investigated in this work is $\sim 50 \%$, but PL QYs as high as $80 \%$ have already been reported for similar structures [36]. It is thus clear that the use of type-II HNCs in LSCs may offer sizable benefits with respect to other commonly used luminophores. It remains, however, to be investigated whether these colloidal type-II HNCs can be easily dispersed into polymer matrices without loss of efficiency.

\section{Acknowledgements}

This work is part of the Joint Solar Programme (JSP) of Hyet Solar and the Stichting voor Fundamenteel Onderzoek der Materie
FOM, which is part of the Netherlands Organisation for Scientific Research (NWO).

\section{Appendix A. Supplementary data}

Supplementary data associated with this article can be found in the online version at http://dx.doi.org.10.1016/j.solmat.2012.12.028.

\section{References}

[1] W.H. Weber, J. Lambe, Luminescent greenhouse collector for solar radiation, Applied Optics 15 (1976) 2299-2300.

[2] A. Goetzberger, W. Greube, Solar energy conversion with fluorescent collectors, Applied Physics 14 (1977) 123-139.

[3] M. Currie, J. Mapel, T. Heidel, S. Goffri, M. Baldo, High-efficiency organic solar concentrators for photovoltaics, Science 321 (2008) 226-228.

[4] M.G. Debije, P.P.C. Verbunt, Thirty years of luminescent solar concentrator research: solar energy for the built environment, Advanced Energy Materials 2 (2012) 12-35.

[5] D. Chemisana, Building integrated concentrating photovoltaics: a review Renewable and Sustainable Energy Reviews 15 (2011) 603-611.

[6] D.J. Farrell, W.G.J.H.M. van Sark, S.T. Velthuijsen, R.E.I. Schropp, Using amorphous silicon solar cells to boost the viability of luminescent solar concentrators, Physica Status Solidi (c) 7 (2010) 1045-1048.

[7] E. Klampaftis, D. Ross, K.R. McIntosh, B.S. Richards, Enhancing the performance of solar cells via luminescent down-shifting of the incident spectrum: a review, Solar Energy Materials and Solar Cells 93 (2009) 1182-1194.

[8] L.H. Slooff, E.E. Bende, A.R. Burgers, T. Budel, M. Pravettoni, R.P. Kenny, E.D. Dunlop, A. Büchtemann, A luminescent solar concentrator with $7.1 \%$ power conversion efficiency, Physica Status Solidi (RRL)-Rapid Research Letters 2 (2008) 257-259.

[9] J.C. Goldschmidt, M. Peters, A. Bösch, H. Helmers, F. Dimroth, S.W. Glunz, G. Willeke, Increasing the efficiency of fluorescent concentrator systems, Solar Energy Materials and Solar Cells 93 (2009) 176-182.

[10] J. Roncali, F. Garnier, Photon-transport properties of luminescent solar concentrators: analysis and optimization, Applied Optics 23 (1984) 2809-2817.

[11] H. Reidenbach, F. Bodem, Optical transmission of bulk plastic material and plastic lightguides at high optical powers, Optics \& Laser Technology 7 (1975) 131-133.

[12] W.G.J.H.M. van Sark, K.W. Barnham, L.H. Slooff, A.J. Chatten, A. Büchtemann, A. Meyer, S.J.Mc. Cormack, R. Koole, D.J. Farrell, R. Bose, E.E. Bende, A.R. Burgers, T. Budel, J. Quilitz, M. Kennedy, T. Meyer, C.D.M. Donegá, A. Meijerink, D. Vanmaekelbergh, Luminescent solar concentrators-a review of recent results, Optics Express 16 (2008) 21773-21792.

[13] A. Hermann, Luminescent solar concentrators-a review, Solar Energy 29 (1982) 323-329.

[14] C. de Mello Donegá, Synthesis and properties of colloidal heteronanocrystals Chemical Society Reviews 40 (2011) 1512-1546.

[15] L. Qu, Z. Peng, X. Peng, Alternative routes toward high quality CdSe nanocrystals, Nano Letters 1 (2001) 333-337.

[16] S. Kim, B. Fisher, H.-J. Eisler, M. Bawendi, Type-II quantum dots: CdTe/ $\mathrm{CdSe}$ (core/shell) and CdSe/ZnTe(core/shell) heterostructures, Journal of the American Chemical Society 125 (2003) 11466-11467.

[17] W. Zhang, G. Chen, J. Wang, B.-C. Ye, X. Zhong, Design and synthesis of highly luminescent near-infrared-emitting water-soluble CdTe/CdSe/ZnS core/shell/ shell quantum dots, Inorganic Chemistry 48 (2009) 9723-9731.

[18] W.G.J.H.M. van Sark, C. De Mello Donegá, R.E. Schropp, Optimizing quantum dot solar concentrators with thin film solar cells, Advances in Science and Technology 74 (2010) 176-181.

[19] R. Kubin, A. Fletcher, Fluorescence quantum yields of some rhodamine dyes, Journal of Luminescence 27 (1983) 455-462.

[20] G. Seybold, G. Wagenblast, New perylene and violanthrone dyestuffs for fluorescent collectors, Dyes and Pigments 11 (1989) 303-317.

[21] M.G. Hyldahl, S.T. Bailey, B.P. Wittmershaus, Photo-stability and performance of CdSe/ZnS quantum dots in luminescent solar concentrators, Solar Energy 83 (2009) 566-573.

[22] M. Debije, P. Verbunt, P. Nadkarni, S. Velate, K. Bhaumik, S. Nedumbamana, B. Rowan, B. Richards, T. Hoeks, Promising fluorescent dye for solar energy conversion based on a perylene perinone, Applied Optics 50 (2011) 163-169.

[23] L.R. Wilson, B.S. Richards, Measurement method for photoluminescen quantum yields of fluorescent organic dyes in polymethyl methacrylate for luminescent solar concentrators, Applied Optics 48 (2009) 212-220.

[24] S. Chandra, J. Doran, S. McCormack, M. Kennedy, A. Chatten, Enhanced quantum dot emission for luminescent solar concentrators using plasmonic interaction, Solar Energy Materials and Solar Cells 98 (2012) 385-390.

[25] A. Efros, A. Efros, Interband absorption of light in a semiconductor sphere, Soviet Physics Semiconductors 16 (1982) 772-775.

[26] A. Chatten, K. Barnham, B. Buxton, N. Ekins-Daukes, M. Malik, Quantum dot solar concentrators, Semiconductors 38 (2004) 909-917. 
[27] C. de Mello Donegá, S.G. Hickey, S.F. Wuister, D. Vanmaekelbergh, A. Meijerink, Single-step synthesis to control the photoluminescence quantum yield and size dispersion of CdSe nanocrystals, The Journal of Physical Chemistry B 107 (2003) 489-496.

[28] J. Bomm, A. Büchtemann, A. Fiore, L. Manna, J.H. Nelson, D. Hill, W.G.J.H.M. van Sark, Fabrication and spectroscopic studies on highly luminescent $\mathrm{CdSe} / \mathrm{CdS}$ nanorod polymer composites, Beilstein Journal of Nanotechnology 1 (2010) 94-100.

[29] S. Gallagher, B. Norton, P. Eames, Quantum dot solar concentrators: electrica conversion efficiencies and comparative concentrating factors of fabricated devices, Solar Energy 81 (2007) 813-821.

[30] S. Gallagher, B. Rowan, J. Doran, B. Norton, Quantum dot solar concentrator: device optimisation using spectroscopic techniques, Solar Energy 81 (2007) 540-547.

[31] P.T.K. Chin, C. de Mello Donegá, S.S. van Bavel, S.C.J. Meskers, N.A.J.M. Sommerdijk, R.A.J. Janssen, Highly luminescent CdTe/CdSe colloidal heteronanocrystals with temperature-dependent emission color, Journal of the American Chemical Society 129 (2007) 14880-14886.

[32] D. Steiner, D.Dorfs.U. Banin, F. Della Sala, L. Manna, O. Millo, Determination of band offsets in heterostructured colloidal nanorods using scanning tunneling spectroscopy, Nano Letters 8 (2008) 2954-2958.
[33] H. Zhong, G.D. Scholes, Shape tuning of type II CdTe/CdSe colloidal nanocrystal heterostructures through seeded growth, Journal of the American Chemical Society 131 (2009) 9170-9171.

[34] R. Koole, M.M. van Schooneveld, J. Hilhorst, C. de Mello Donegá, D.C. 't Hart, A. van Blaaderen, D. Vanmaekelbergh, A. Meijerink, On the incorporation mechanism of hydrophobic quantum dots in silica spheres by a reverse microemulsion method, Chemistry of Materials 20 (2008) 2503-2512.

[35] A.L. Efros, M. Rosen, M. Kuno, M. Nirmal, D.J. Norris, M. Bawendi, Band-edge exciton in quantum dots of semiconductors with a degenerate valence band: dark and bright exciton states, Physical Review B 54 (1996) 4843-4856.

[36] C. de Mello Donegá, Formation of nanoscale spatially indirect excitons: evolution of the type-ii optical character of CdTe/CdSe heteronanocrystals, Physical Review B 81 (2010) 165303.

[37] C. de Mello Donegá, R. Koole, Size dependence of the spontaneous emission rate and absorption cross section of CdSe and CdTe quantum dots, The Journal of Physical Chemistry C 113 (2009) 6511-6520.

[38] A. Zastrow, Physics and applications of fluorescent concentrators: a review, Proceedings of SPIE-The International Society for Optical Engineering 2255 (1994) 534-547. 